\title{
A novel and effective ophthalmology morbidity and mortality meeting
}

\author{
K. L. Chan ${ }^{1} \cdot$ J. Deutsch ${ }^{1} \cdot$ G. Morphis ${ }^{1} \cdot$ S. Scotcher ${ }^{1} \cdot$ A. Smith ${ }^{1} \cdot$ B. While ${ }^{1} \cdot$ S. Madge ${ }^{1}$
}

Received: 9 August 2018 / Accepted: 10 August 2018 / Published online: 7 September 2018

(c) The Royal College of Ophthalmologists 2018

Morbidity and mortality (M\&M) meetings drive learning from clinical outcomes and improve service delivery in surgery [1]. They are regular scheduled meetings that discuss adverse surgical outcomes for patients and were originally designed for surgical training. M\&M meetings are common place in other surgical specialties and are recommended by the Royal College of Surgeons as part of maintaining and improving patient safety [2]. However, M\&M practice appears to be lacking in UK ophthalmology with no recommendations from the Royal College of Ophthalmologists or research into its potential benefits. We present a novel method of integrating M\&M meetings to ophthalmology practice which has been running in our department since November 2016. We also present a qualitative analysis of its impact on the department since its inception.

To collect M\&M incidents, we took a simple twopronged approach: a 'glitchbook' was placed in the ophthalmic theatre to prospectively capture at source any surgical complications; in addition, an email address was created to capture $M \& M$ in the clinic setting. In both methods, incidents are reported by the surgeon and/or any auxiliary member of the team.

The M\&M meeting was integrated into the departments by two monthly audit meetings to reduce the administrative burden of a separate M\&M meeting. These meetings included the wider multidisciplinary team: consultants, trainees, nursing staff and auxiliary staff. Each incident collected is presented by the operating surgeon, which can be in the form of a short description of events, through to a full presentation with a video of the operation. Following the presentations of the case, an open and

S. Madge

Simon.Madge@wvt.nhs.uk

1 Victoria Eye Unit, Hereford County Hospital, Hereford HR1 2ER Herefordshire, UK reflective discussion about the surgical issues occur with involvement of the whole team. In each case, learning points and reflections are noted and any changes needed are implemented and discussed at the next meeting.

A qualitative analysis involving semi-structured interviews and questionnaires was performed to investigate the impact of M\&M meetings on the department. There were multiple themes that arose from this study: training and education, cultural change and improving patient care. All surgeons from a trainee to a consultant felt that this was an excellent opportunity and resource for learning. The trainees particularly felt the benefit from experienced feedback from multiple consultants and the chance to hone their presentation skills. All members of the staff felt that the M\&M meetings had fostered a blame-free and open culture within the department. The combination of discussing M\&M issues and open culture was felt to be strongly driving improvements in patient safety.

Our departments experience of M\&M meetings is that we have found it an easy process to implement, with great benefits for training and patient safety. We recommend that UK ophthalmology keeps up with the other specialties and adopts $\mathrm{M} \& \mathrm{M}$ as a standard practice.

\section{Compliance with ethical standards}

Conflict of interest The authors declare that they have no conflict of interest.

\section{References}

1. Good Surgical Practice-Royal College of Surgeons. https://www. rcseng.ac.uk/standards-and-research/gsp.

2. Morbidity and Mortality Meetings-Royal College of Surgeons. https://www.rcseng.ac.uk/standards-and-research/standards-andguidance/good-practice-guides/morbidity-and-mortality-meetings 